\title{
Data Tectonics - a framework for Building Physical and Immersive Data Representations
}

\author{
Carmen Hull
}

Wesley Willett

\section{Thinking Like an Architect}

The emerging research areas of physical [1], immersive [2], and situated [3][4] data representations promise to revolutionize our experience of data - moving data visualizations off of the desktop and instead surfacing data into the world we inhabit, touch, and sense with our whole bodies. While full of opportunities, this shift also poses immense new challenges for visualization designers, who must now contend with the reality of creating systems that have the spatiality, materiality, and scale of real-world environments. While the visualization community has long considered the role of perception and cognition for screen- and paperbased visualizations, designers of data physicalizations and situated visualizations must now consider our perception and experience of physical environments, material properties, cultural symbolism, and spatial relationships.

Fortunately, many of the challenges posed by these new data representations echo those which architects have long faced when designing the built environment. Architects use the term "tectonics" to characterize the holistic process of reconciling the various competing factors inherent in challenging design problems. We extend this term to include the concept of "data tectonics" to describe the integrative practices surrounding the designing of complex, embodied, and data-driven representations. This preliminary discussion explores new ways of thinking about data representations, inspired by two long-standing practices used in architectural design - diagramming and modeling. Our discussion facilitates a deeper understanding of the structure and materiality of data representations.

\section{Architectural Tectonics}

Most of us are familiar with the idea of tectonics in relation to geographic plates, as the forces involved in plate tectonics and the structural features resulting from them [5]. The term is derived from the ancient Greek "tektonikos", meaning "relating to construction or building" [6]. The original use of the word, therefore, was architectural, and it is still commonly used in architectural education and practice to describe the relationship between the built form, technology, and culture.

Tectonics in architecture serves as an integrative theory that examines "the interwoven relationship between space, function, structure, context, symbolism, representation and construction" [7]. It is a systemic way of thinking about specific contemporary and local conditions, which in turn influence production methods, construction and materials. Tectonics implies not only a constructional logic, but also "material realities that reveal narrative meaning" [8]. For example, the tectonics of a traditionally hand-built Japanese pagoda (Figure 1) are starkly different in origin and purpose from a digitally fabricated parametrically designed building (Figure 2). Japanese joinery is a sophisticated, pre-industrial system for creating large hand-made timber frame structures. The intricate tectonic system of joints, proportions, 
context and symbolism allows for a variety of spatial arrangements and styles through a handful of techniques, with each region featuring unique characteristics and variations [9].



Figure 1. Traditional joinery techniques used in vernacular Japanese architecture. Image permission pending.

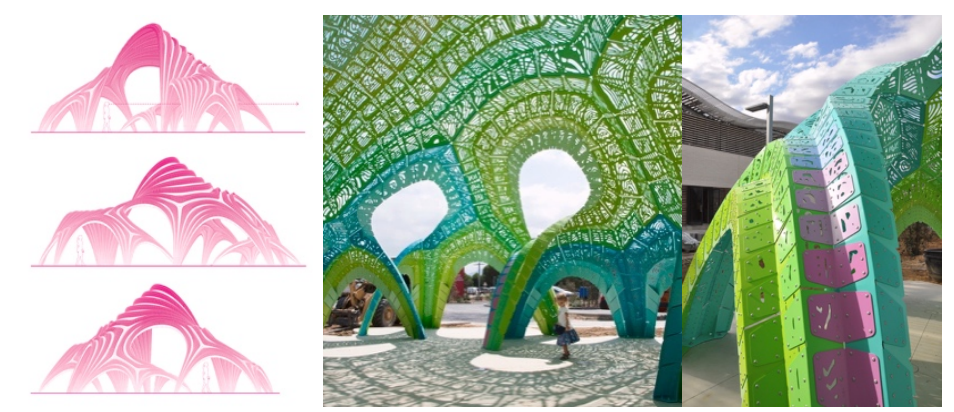

Figure 2. Pavilion - Marc Fornes. Image permission pending.

In contrast, the pavilion in Figure 2, employs a radically different tectonic approach through the use of computation and digital fabrication. Both methods reflect the technology, culture, and context of a specific time and place. There is a transparency to the constructional and material logic of each building that communicates meaning, provenance, affordances, and function. When these pieces are put together cohesively, they communicate "the poetic integration of assembly, materiality, representation, space and environment" [10].

\section{Digital Tectonics and Data Tectonics}

The concept of tectonics continues to inspire architects in the shift to digital design and fabrication. The pavilion shown in Figure 2, was designed using a dynamic computational approach that is heavily influenced by parameterization and generative design tools. Often, contemporary architects no longer merely envision a form and build it. Instead, they are increasingly integrating computation into the act of design, crafting rules, constraints, and primitives that can combine together to produce unexpected, emergent forms based on the parameters of geometry, fabrication and context. Architect Neil Leach and others describe these emergent structuring principles as "digital tectonics" - adapting the operations of intelligent self-organizing systems such as ant behavior, swarms and urban growth or 'small components working together' to computational processes [11]. 


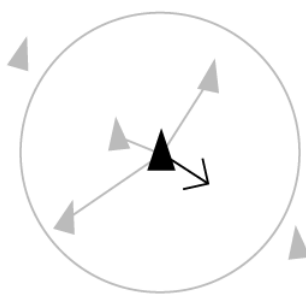

Separation:

Steer to avoid crowding

local flockmates

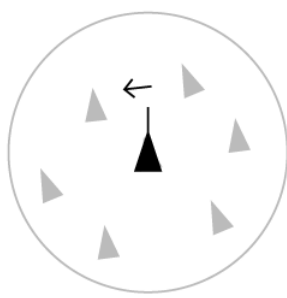

Alignment:

Steer toward the average heading of local flockmates

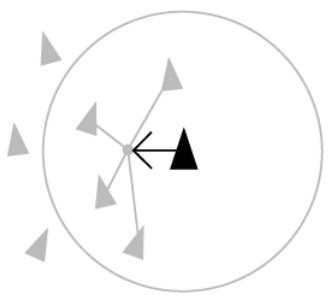

Cohesion:

Steer to move toward the average position of local flockmates

Figure 3. Boids Behavior Diagrams.

As shown in Figure 3, dynamic processes such as the pattern of birds in flight (or 'Boids', the computational equivalent) display a structural logic that has no physical connections between the individual data points, however the rules of each agent create an emergent sense of cohesion.

When creating a traditional structure (Figure 1), the tectonics of the repeated pattern of wooden joints and structural forms are composed together to create a spatial configuration we recognize (in this case a "Japanese pagoda"). The same operations can be repeated somewhere else in a slightly different way to create a new "Japanese pagoda" object that suits a different set of local conditions. Conceptually, Marc Fornes' pavilion (Figure 2) also uses a tectonic approach which focuses on the relationship between the physical components, but generates the geometry and connections using computation and digital fabrication methods to produce a very different final product.

In summary, traditional tectonics focuses on the physical relationships between the various components of a building that create a cohesive whole. Digital tectonics uses this concept to inspire a bottom-up computational process of repetitive, localized operations to generate new geometrical formations, often based on material properties or digital fabrication methods.

Data tectonics further extends these concepts to the making of physical and immersive visualizations, to emphasize the need for integration between data, structure, material and fabrication method and to point to techniques that can clarify these relationships. 


\section{Tectonics in Data Visualization Design}

The design process in architecture often revolves around artifact-centered creation practices, including diagramming and modeling, that help architects unpack and interrogate the tectonics of their designs. Already, visualization and physicalization designers may benefit from adopting diagramming and modelling approaches. In fact, tools like the five-design sheet method [12] and constructive visualization toolkits [13] are already a small step in this direction. However, considering the tools and literature around tectonics in architecture suggests a rich space for new visualization design tools and techniques inspired by architectural diagramming and modelling practices.

In architectural practice, diagrams distill the structure and essential relationships or behavior of a system into a 2D drawing, and models test these relationships through exploration of expressions in material form. By making diagrams and models during the design process to show the relationships between the form and the data, the tectonic language can be articulated and clarified. Figure 4 shows a typical design process in architecture including a) a concept, or "parti" diagram, b) a sectional diagram, c) fabricated test pieces and d) a finished model. In the following section, we examine how diagramming and modeling practices facilitate a deeper understanding of structure and materiality and how they might enable a richer appreciation of the tectonics of physical and virtual data representations. Both approaches have the potential to help visualization designers resolve the divergent elements of a design into one vision.
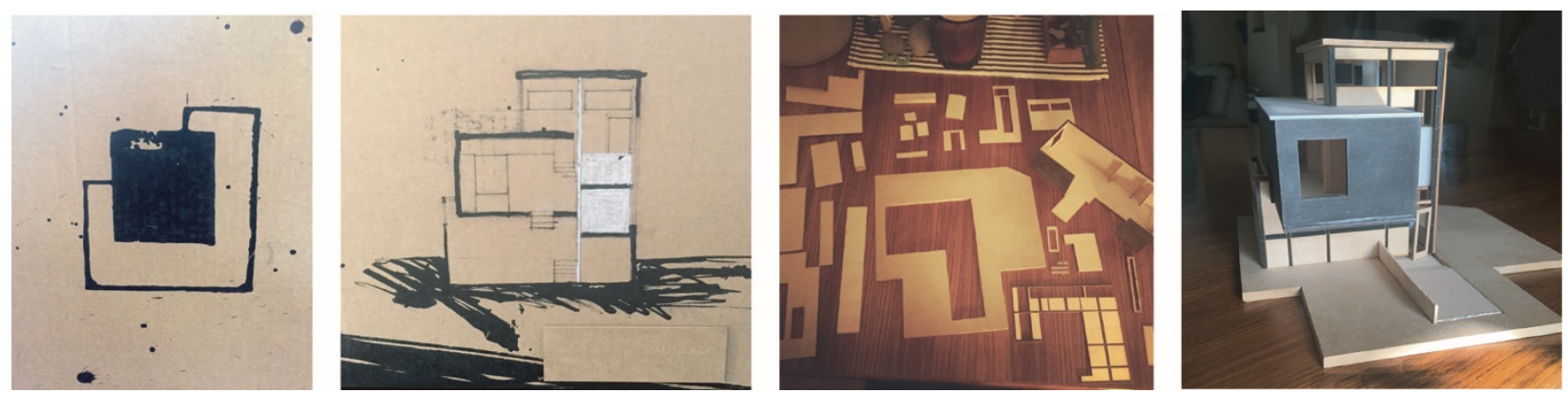

Figure 4. Diagrams and Models used in the Design Process of Architecture. Source: Author

\section{Diagrams}

Architects use diagrams for both synthesis and genesis, with the diagram functioning as evidence of how a concept will work and to "synthesize pictorial and linguistic attributes, or representation and presentation to produce new insight and knowledge" [14]. Ljungberg et al. describe the function of diagrams as "apparatuses of graphic homogenization [that] become the locus in which relations can come into existence" [15]. One of the more powerful potentials for diagrammatic thinking is as a "reproducible schema that can be instantiated in diverse display formats" [15]. The potential of diagrammatic thinking, therefore, lies in its power to reduce a problem or idea to its essence, so that it can become a generative tool for different solutions to a design problem. These types of diagrams can be described as "performative" 
rather than representational [16]. One example of the transferability of diagrammatic thinking between architecture and computation is in the influence of architect Christopher Alexander on agile software development and the wiki engine [17]. As the inventor of "pattern languages", he emphasizes the importance of the practice in his book "Notes on the Synthesis of Form" that "the starting point of synthesis is the diagram" [18]. Thus, diagrams are one tool to help designers clarify and communicate the initial tectonic concepts of a design problem that can then be tested by making models with various different materials and methods

\section{Models}

There is a long history of model making in both science [19] and architecture [20] that can richly inform the making of data physicalizations. Architects use models to prototype, validate and refine their designs. The tectonics of a design is realized in the negotiation between structure and material production, between diagram and model, between how something works and what it looks like. Finnish architect Juhani Pallasmaa talks about the ability of materials to speak a "language" of their own - "stone speaks of its ancient geological origins, inherent symbolism of permanence. Bronze evokes the extreme heat of its manufacture, the process of time as measured in its patina. Wood speaks of its two existences and time scales; its first life as a tree and the second as a human artifact" [21]. Encoding data into the material and physical properties of an object adds an additional layer of meaning that can support a rich experience of the data. Tectonic theory aims to integrate material choices into the structural characteristics of the data and interactions, to unify both the digital and physical aspects of data-driven artefacts. Choosing a material and fabrication method will be based on not only the visual appearance, but also its affordances, characteristics and functionality. In architectural tectonics, for example, a certain cut of lumber is chosen not only for the appearance of a particular grain pattern, but also for its mechanical properties and performance as a structural member. Developing the material choices and fabrication method, or the what and how of a data representation, in tandem with the structural aspects ensures that the physical components are linked to the intrinsic structure of the data.

Data tectonics also implies a degree of craftsmanship that comes from a thorough interaction with a data set and the medium of expression, in both physical and digital realms. We make digital constructions in the same way we make physical constructions of architecture, through repetitive, sequences of small accumulations of actions that build on top of each other. As Malcolm McCullough writes, even digital design "reveals authorship at the level of its internal organization". Some examples he gives of "continuous operations" in digital design are: adjusting lights and cameras for digital rendering, or refining parametric code to generate novel form families, to discover affordances and constraints inherent in the medium [22]. In the following case study, the tectonic concept of layers is reflected in both the structure of the digital data files and the physical components of the representation. The materials, fabrication method, visual representation, interactions and display mechanism all use the language of layers. 
The installation in Figure 5 features a 3D physical campus model visualizing layers of historical photos showing 70 years of campus development, as well as energy use data of individual buildings over the course of a year, to explore connections between age, energy use and building form. The theme of layers was carried through to the display mechanism of a bottom projected illuminated surface, where light is projected up through the model to animate the form with light encoded data. Because the nature of the data we received for the building outlines was individual floor outlines, each individual model was fabricated by laser cutting individual floor plates out of acrylic sheets and assembled by hand. Thus, the format of the original data inspired the theme that was expressed through both digital and material properties in the rest of the components.
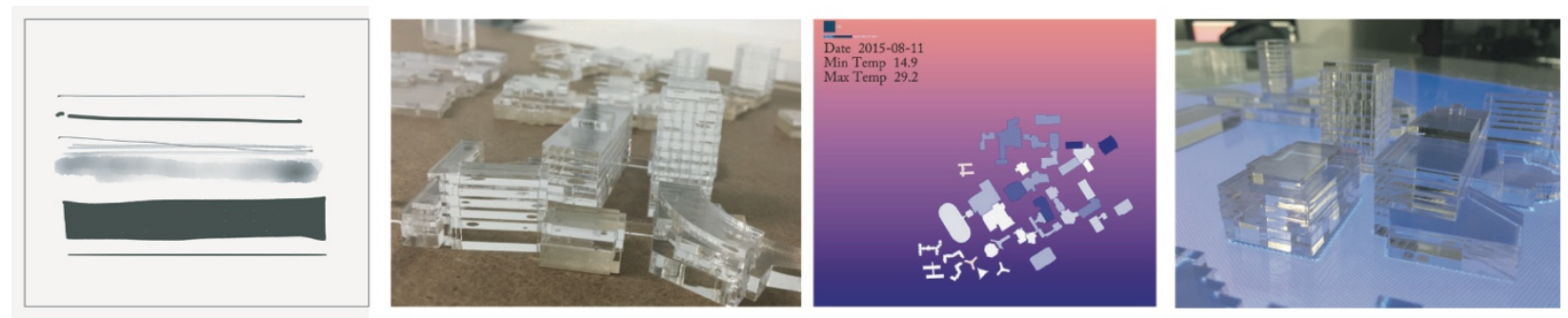

Figure 5. Layers of history, information, material and meaning.

Data designers can use this example to consider the nature of the data they are representing, and integrate the structure and the materials in a way that clearly underscores the qualities and interactions they wish to emphasize. Using multiple diagrams and models in the design process

of this installation allowed the designer to discover similar characteristics between the data and the interactions that would be best expressed through the use of layers. During the design process, other fabrication choices such as 3D printed models or top projected visuals were discarded in favor of those that supported the main idea.

\section{Conclusion}

Physical and immersive data representations are challenging to design and create because of the complexity introduced by physical materials, fabrication techniques, environmental conditions, and physical interactions. Architects use the term "tectonics" (1) to refer to the intangible characteristics of a cohesive design solution, and (2) to characterize the holistic process of reconciling the various competing factors (material properties, cultural symbolism, spatial relationships, etc.) inherent in challenging design problems. Variations of this idea include the notion of "digital tectonics" which reframes these challenges in the context of digital generative design and provide a link on how to apply our concept to the design of digital and physical data representations. 
To confront the intangible challenges inherent in designing new physical and immersive data representations, we propose the concept of "data tectonics" to describe the holistic nature of designing these complex, embodied, and data-driven representations. Data tectonics examines the relationship between context, data, visual representation, materiality, fabrication and interactions of a data representation. Visualization designers can borrow from longstanding approaches used by architects to help confront and reconcile competing design factors. These include diagramming and modeling to extract the essential characteristics of structure and materiality that a data designer wishes to focus on. Both approaches are designed to resolve and integrate divergent elements into one vision, and help designers channel the emerging essence of their vision for a project.

\section{REFERENCES}

[1] Y. Jansen, P. Dragicevic, P. Isenberg, J. Alexander, A. Karnik, J. Kildal, S. Subramanian, and K. Hornbaek, "Opportunities and Challenges for Data Physicalization," Proc. SIGCHI Conf. Hum. Factors Comput. Syst., pp. 3227-3236, 2015.

[2] B. Bach, T. Chandler, M. Cordeil, T. Czauderna, T. Dwyer, J. Glowacki, C. Goncu, M. Klapperstueck, K. Klein, K. Marriott, F. Schreiber, and E. Wilson, "Immersive Analytics," IEEE Transactions on Visualization and Computer Graphics, Sep-2017. [Online]. Available: http://ieeexplore.ieee.org/lpdocs/epic03/wrapper.htm?arnumber=7314296. [Accessed: 17-Sep-2016].

[3] A. Vande Moere and D. Hill, "Designing for the Situated and Public Visualization of Urban Data," J. Urban Technol., vol. 19, no. 2, pp. 25-46, 2012.

[4] W. Willett, Y. Jansen, and P. Dragicevic, "Embedded Data Representations," IEEE Trans. Vis. Comput. Graph., vol. 23, no. 1, pp. 461-470, 2017.

[5] "Merriam-Webster." [Online]. Available: https://www.merriamwebster.com/dictionary/tectonics. [Accessed: 14-Jul-2018].

[6] "Online Etymology Dictionary." [Online]. Available: https://www.etymonline.com/word/tectonic. [Accessed: 14-Jul-2018].

[7] C. J. Schwartz, Introducing Architectural Tectonics: Exploring the Intersection of Design and... / Request PDF. Routledge, 2016.

[8] C. Bundgaard, "Tectonics of montage. Architectural positions for a tectonic sustainable building practice," in Structures and Architecture, CRC Press, 2013, pp. 391-398. 
[9] D. Y. Yen, "Japanese Timber Frame Methodology: Alternative Solutions to Hawaii 's Built Environment Japanese Timber Frame Methodology :," no. May, pp. 1-154, 2012.

[10] C. Schwartz, "Spiritual Tectonics : Exploring Dualities in the Design Studio," in 30th National Conference on the Beginning Design Student: Materiality, Essence + Substance, 2014.

[11] N. Leach, D. (Architect) Turnbull, and C. (Chris J. K. . Williams, Digital tectonics. WileyAcademy, 2004.

[12] J. C. Roberts, C. Headleand, and P. D. Ritsos, "Sketching Designs Using the Five Design-Sheet Methodology," IEEE Trans. Vis. Comput. Graph., vol. 22, no. 1, pp. 419428, 2016.

[13] S. Huron, Y. Jansen, and S. Carpendale, "Constructing visual representations: Investigating the use of tangible tokens," IEEE Trans. Vis. Comput. Graph., vol. 20, no. 12, pp. 2102-2111, 2014.

[14] E. Y. L. Do and M. D. Gross, "Thinking with diagrams in architectural design," Artif. Intell. Rev., vol. 15, no. 1-2, pp. 135-149, 2001.

[15] C. Ljungberg, "Thinking and diagrams - An introduction," S. Krämer and C. Ljungbert, Eds. De Gruyter, Inc., 2018, pp. 1-20.

[16] A. Vidler, "Diagrams of Diagrams: Architectural Abstraction and Modern Representation," Press. Calif., vol. 72, no. 72, pp. 1-20, 2009.

[17] W. Cunningham and S. Foundation, "Wiki as Pattern Language," Proc. 20th Conf. Pattern Lang. Programs, 2013.

[18] C. Alexander, Notes on the synthesis of form. Harvard University Press, 1964.

[19] S. de. Chadarevian and N. Hopwood, Models : the third dimension of science. Stanford University Press, 2004.

[20] C. Hull and W. Willett, "Building with Data : Architectural Models as Inspiration for Data Physicalization," in Proceedings of the 2017 CHI Conference on Human Factors in Computing Systems (2017), 2017, pp. 1217-1264.

[21] J. Pallasmaa, "Hapticity and Time," in Encounters 1 - Architectural Essays, Rakennustieto Publishing, 2013. 
[22] M. McCullough, Digital Ground: Architecture, Pervasive Computing, and Environmental Knowing. MIT Press, 2004. 